\title{
Endoscopic ultrasound-guided pancreatico- gastrostomy using a lumen-apposing metal stent plus a double-pigtail plastic stent
}
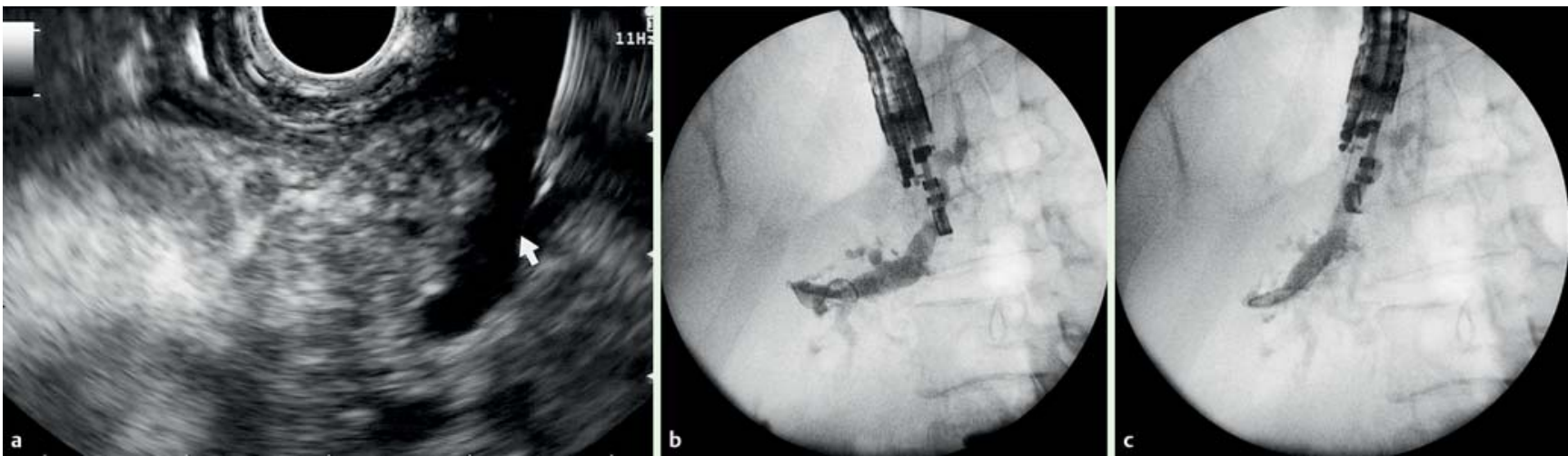

Fig. 1 Endoscopic ultrasound (EUS)-guided access to the pancreatic duct. a Transgastric puncture of the dilated pancreatic duct using a 19-gauge needle (Expect Flex; Boston Scientific, Marlborough, Massachusetts, USA). b EUS-guided pancreatography revealed a dilated, tortuous main pancreatic duct, and severe stricture in the cephalic pancreatic area. A 0.035 -inch guidewire was advanced through the pancreatic duct. c Fluoroscopic view of the $6 \mathrm{Fr}$ cystotome over the guidewire.
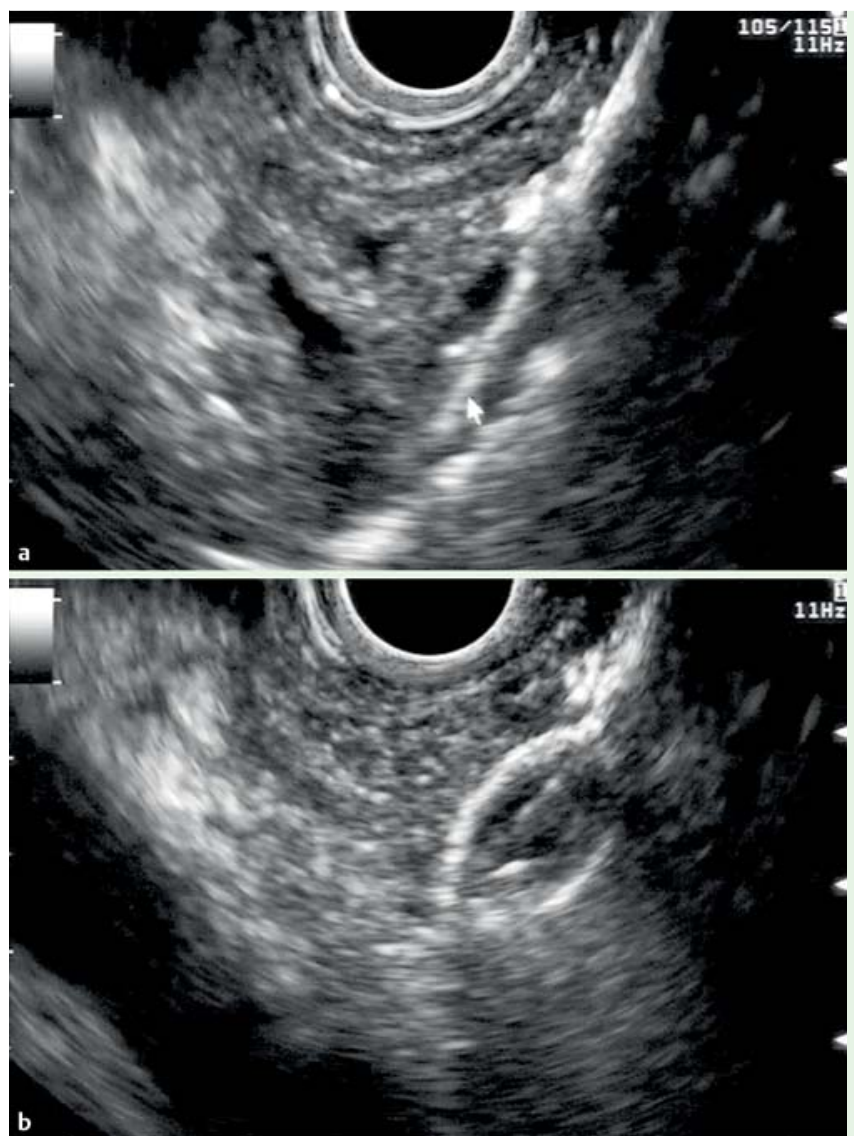

Fig. 2 Placement of a lumen-apposing metal stent (LAMS). a Endoscopic ultrasound (EUS) image of the HotAXIOS catheter (9Fr; Boston Scientific, Marlborough, Massachusetts, USA), energized and inserted inside the pancreatic duct. $\mathbf{b}$ EUS-guided deployment of the distal end of the LAMS ( $6 \times 8 \mathrm{~mm}$, HotAXIOS). Image shows the LAMS distal flange located inside the pancreatic duct.

Endoscopic ultrasound (EUS)-guided pancreatic duct drainage remains one of the most technically challenging endosonography interventions. There is a lack of specific devices for the technique, and the question of which kind of stent should be used remains controversial: plastic (straight, single or double pigtail) or selfexpanding metal [1-4].

The lumen-apposing metal stent (LAMS) has been used in different scenarios (transmural drainage of collections and

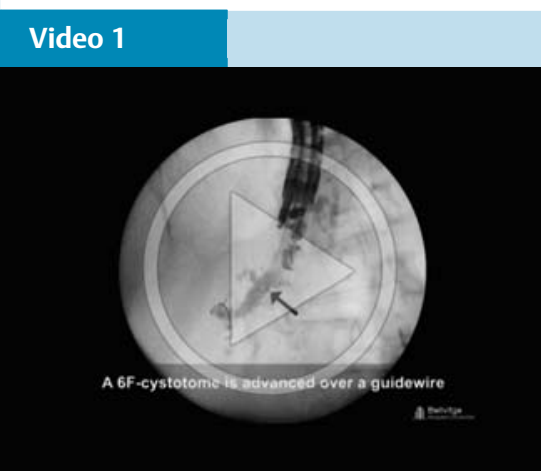

Endoscopic ultrasound-guided transluminal pancreatic duct drainage (pancreaticogastrostomy) using a lumen-apposing stent plus a double-pigtail plastic stent.

EUS-guided anastomosis). There are various sizes of LAMS available, and some of them, specifically designed to be used in the bile duct, are small (e.g. $6 \times 8 \mathrm{~mm}$ ) [5]. To our knowledge, this is the first report to date of successful EUS-guided pancreatic duct drainage using a LAMS plus a pigtail stent. A 44-year-old man, with chronic pancreatitis and pancreatic duct stricture causing abdominal pain, was referred to our unit. Endoscopic retrograde cholangiopancreatography was attempted, but cannulation was unsuccessful. After a failed rendezvous, EUS-guided transluminal pancreatic duct drainage (pancreaticogastrostomy) was successfully performed using a biliary LAMS $(6 \times 8 \mathrm{~mm}$, HotAXIOS; Boston Scientific, Marlborough, Massachusetts, USA) plus a double- 


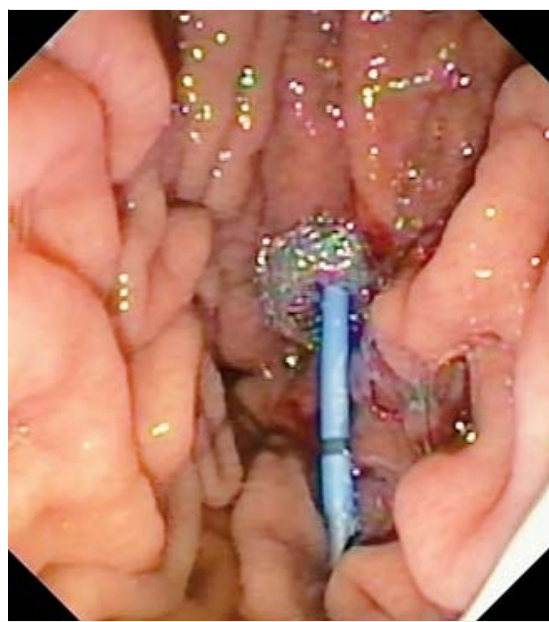

Fig. 3 Endoscopic view of a double-pigtail plastic stent $(7 \mathrm{Fr} \times 5 \mathrm{~cm}$, Advanix; Boston Scientific, Marlborough, Massachusetts, USA) through the lumen-apposing metal stent in the gastric cavity.

pigtail stent, with the purpose of avoiding self-occlusion, food impaction, dislodgement, and migration ( $\bullet$ Video 1 ).

First, the dilated pancreatic duct (up to $5.6 \mathrm{~mm}$ ) was punctured directly from the gastric wall, using a $19 \mathrm{G}$ needle, and tract dilation was carried out using a $6 \mathrm{Fr}$ cystotome over a 0.035 -inch guidewire ( $\bullet$ Fig. 1). Second, a LAMS was inserted and deployed using the HotAXIOS system. All four steps of the delivery system were performed under EUS, endoscopic, and fluoroscopic guidance ( $\bullet$ Fig. 2). Finally, a double-pigtail plastic stent $(7 \mathrm{Fr} \times 5 \mathrm{~cm}$, Advanix; Boston Scientific) was advanced through the LAMS under endoscopic vision ( Fig.3). The total procedure duration was 48 minutes. The patient's condition evolved satisfactorily without any adverse events.

The use of a LAMS plus a double-pigtail stent in EUS-guided pancreatic duct drainage was technically feasible and safe, and reduced the potential risk of pancreatic fluid leak or stent migration. For these reasons, it should be considered as a new option in this scenario.

Endoscopy_UCTN_Code_TTT_1AS_2AD

Competing interests: None

Joan B. Gornals ${ }^{1}$, Claudia Consiglieri ${ }^{1}$, Francesc Vida ${ }^{2}$, Carme Loras ${ }^{3}$

${ }^{1}$ Endoscopy Unit, Department of Digestive Diseases, Hospital Universitari de Bellvitge-IDIBELL, Barcelona, Spain

2 Endoscopy Unit, Department of Digestive Diseases, Althaia Xarxa Hospitalaria de Manresa, Manresa, Spain

${ }^{3}$ Endoscopy Unit, Department of Digestive Diseases, Hospital Universitari Mutua de Terrassa, CIBERehd, Spain

\section{References}

1 Chen YI, Saxena P, Ngamruengphong $S$ et al. Endoscopic ultrasound-guided pancreatic duct drainage: technical approaches to a challenging procedure. Endoscopy 2016; 48: $192-193$

2 Chapman CG, Waxman I, Siddiqui UD. Endoscopic ultrasound (EUS)-guided pancreatic duct drainage: the basics of when and how to perform EUS-guided pancreatic duct interventions. Clin Endosc 2016; 49: 161 - 167

3 Fujii-Lau LL, Levy MJ. Endoscopic ultrasound-guided pancreatic duct drainage. J Hepatobiliary Pancreat Sci 2015; 22: 51 - 57

4 Will U, Reichel A, Fueldner F et al. Endoscopic ultrasonography-guided drainage for patients with symptomatic obstruction and enlargement of the pancreatic duct. World J Gastroenterol 2015; 21: 13140-13151

5 Gornals JB, Consiglieri C, Gallarreta $V$ et al. Single-session fluoroless endoscopic ultrasound-guided fine-needle aspiration and choledochoduodenostomy with a biliary lumen-apposing stent. Endoscopy 2015; 47: $418-419$

Bibliography

DOI http://dx.doi.org/

10.1055/s-0042-113186

Endoscopy 2016; 48: E276-E277

(c) Georg Thieme Verlag KG

Stuttgart · New York

ISSN 0013-726X

\section{Corresponding author}

Joan B. Gornals, MD, PhD

Endoscopy Unit

Department of Digestive Diseases

Hospital Universitari de Bellvitge-IDIBELL

(Bellvitge Biomedical Research Institute)

Feixa Llarga s/n

08907 L'Hospitalet de Llobregat

Barcelona

Catalonia

Spain

Fax: +34-93-2607681

jgornals@bellvitgehospital.cat 